\section{PELVIC TUBERCULOSIS AND PREGNANCY}

BY

\section{IAN A. DONALDSON, F.R.C.S., M.R.C.O.G First Assistant in Gynaecology and Obstetrics, St. George's Hospital, London}

The problem of pregnancy in relation to pelvic tuberculosis is receiving increasing attention. Two patients seen recently illustrate some aspects of this question. The first was known to have pelvic tuberculosis, the diagnosis being made at laparotomy. An intrauterine pregnancy began and spontaneous abortion occurred. The second patient had an uneventful pregnancy, labour, and puerperium. The infant was healthy and has remained so. Six months after confinement treatment was sought for dyspareunia. Tender adnexal swellings were found. Bilateral pyosalpinges and an intramural abscess of the right cornu of the uterus were removed at laparotomy. These proved to be tuberculous.

\section{Case 1}

On September 2, 1946, the patient attended the hospital. She was 23 years old and had been married two years. She had four symptoms: pain in lower abdomerı and back for nine months; excessive loss at the periods; sterility of two years' duration ; and painful intercourse for nine months. The only physical abnormality detected was an ill-defined tender swelling in the left fornix. On September 8 she was admitted for examination under anaesthesia. This revealed a prolapsed left ovary but no other pelvic abnormality. $\mathrm{Re}-$ examination without anaesthesia confirmed the deep tenderness on the left side, and operation was advised to cure the dyspareunia.

Laparotomy was performed on September 11. On opening the abdomen it was found that the posterior surface of the uterus, the ovaries, the Fallopian tubes, and the posterior surface of the broad ligaments were studded with tiny tubercles. A portion of the omentum was similarly affected and adherent to the parietal peritoneum ; part of the omentum was removed and sent for pathological examination. Both ovaries were prolapsed. The ovarian ligaments were shortened and ventrosuspension was performed. The pathological report stated that the omentum was very congested, possibly owing to operative trauma. Convalescence was uneventful, and the patient was discharged on September 29. She attended the follow-up clinic on November 11, by which time the pain and dyspareunia had ceased, the uterus was well anteverted, and there appeared to be no indication for further treatment.

On May 19, 1948, she was admitted as an emergency case, complaining of attacks of lower abdominal pain and shivering for the past three days, pain on passing urine, and heavy losses at the periods. On admission the temperature was $103^{\circ} \mathrm{F}$. $\left(39.4^{\circ} \mathrm{C}\right.$.). Bilateral tender tubal swellings were found on pelvic examination. A diagnosis of salpingitis was made and chemotherapy begun. The white-cell count was 20,000 per c.mm. (polymorphs $83 \%$, lymphocytes $12 \%$, monocytes $5 \%$ ). The pyrexia slowly subsided during the next five days. On May 27 the temperature was normal. The bilateral tubal swellings were larger and extended about 3 in. $(7.5 \mathrm{~cm}$.) above the pubis. The white-cell count was 11,200 per c.mm. On June 24 the general condition was satisfactory. The white-cell count was 8,400 per c.mm.; the pelvic swelling was much smaller. Short-wave diathermy to the pelvis was begun. The patient was discharged on June 12. On July 19 she attended the follow-up clinic There were no symptoms, but on pelvic examination a tender tubal swelling was still present behind the.uterus, and laparotomy was advised.

On August 15 she was readmitted. A pre-operative course of chemotherapy was started. Laparotomy, performed on
August 23, revealed extensive intra-abdominal adhesions between the omentum, intestines, uterus, Fallopian tubes, and bladder, and multiple fine tubercles on the pelvic viscera. The right Fallopian tube was grossly enlarged and surrounded by dense adhesions. The uterus was slightly enlarged, as was the left Fallopian tube. The adhesions were so dense that no plane of cleavage could be found. A piece of omentum removed for histological examination showed numerous typical giant-cell systems scattered throughout the tissue. On August 24 a small haemoptysis occurred. Radiological examination of the lungs revealed an area in the right mid-zone suggestive of bronchiectasis. The patient was transferred to the chest unit on August 28. The erythrocyte sedimentation rate was raised and slowly fell to normal. The sputum was repeatedly examined, but no tubercle bacilli were found. Periodical radiological examination of the lungs revealed no abnormality, the earlier shadow having vanished. A low-grade pyrexia continued, and abdominal and pelvic pain persisted.

A course of streptomycin, $0.5 \mathrm{~g}$. twice daily, was begun on November 25. The patient had not received streptomycin previously. The course was completed on January 5,1949 ; a total dosage of $30 \mathrm{~g}$. was given.

Gynaecological examination on January 17 revealed a mass approximately 3 to $4 \mathrm{in}$. $(7.5$ to $10 \mathrm{~cm}$.) in diameter in the region of the right ovary; no other abnormality was detected.

The patient remained under observation and gradual resumption of activity was allowed. She was discharged from the chest unit on March 5.

On November 23 she attended the orthopaedic department because she had developed pain and stiffness in the left shoulder. Arthrodesis of the left shoulder was performed for caries sicca in December, 1949. At a further operation on the same joint in November, 1950, a portion of the capsule was excised and examined histologically. This confirmed tuberculosis of the shoulder-joint. The patient remained under the care of the orthopaedic followup clinic.

On May 21, 1951, she attended the gynaecological outpatient department. Her last menstrual period had begun on March 15; her periods had been regular until then. On May 15 she had started to bleed. She had been seen at another hospital and had been told that she was pregnant. In view of her history pregnancy seemed a most unlikely diagnosis. The uterus was enlarged and softened, but did not feel as soft as a normal healthy pregnant uterus. A pregnancy test (Geist-Salmon-Frank) was performed to satisfy the patient, who was desperately keen to have a child. The pregnancy test was positive.

The patient was admitted on May 29. Since last seen she had had more vaginal bleeding, and this had been accompanied by lower abdominal pain. The pelvic physical signs had not changed. Doubt was thrown on the result of the pregnancy test ; this was repeated, but was again positive. The patient was treated for threatened abortion. On June 14 a further pelvic examination revealed no change in the pelvic signs. A further pregnancy test was positive.

On June 22 the patient aborted, typical chorio-decidual tissue being passed. Evacuation of the uterus was performed on June 25 ; a small amount of chorio-decidual tissue was obtained. Post-operatively, blood transfusion was given. Convalescence was otherwise uneventful. She was discharged on July 8 .

The patient attended the follow-up clinic on July 30 ; she was then in good health. The pregnancy test was repeated and found to be negative. The patient has been advised to attend periodically for assessment.

\section{Case 2}

This patient, aged 22, was first seen on March 14, 1950, at the antenatal clinic; this was her first pregnancy. She was three months pregnant, obstetrically straightforward, and gave no history of any serious illness in the past. 
Spontaneous delivery of a full-term normal female infant occurred on September 22. The birth weight was $5 \mathrm{lb} .9 \mathrm{oz}$. $(2.5 \mathrm{~kg}$.$) , and the puerperium was normal. On November 1$ she attended the post-natal clinic and no abnormality was detected apart from a slight greenish vaginal discharge. On November 29 the vaginal discharge was mucoid.

On March 14, 1951, the patient came to the post-natal clinic. Her symptoms were dyspareunia for a month and lower abdominal pain, like period pains, since January. The periods had recommenced in February. Pelvic examination showed a slight greenish mucopurulent discharge and tender bilateral adnexal swellings thought to be enlarged prolapsed ovaries.

She was admitted on April 2, when the only additional history obtained was of severe dysmenorrhoea since 1948. Laparotomy was performed on April 4. Bilateral pyosalpinges, an intramural abscess at the right cornu of the uterus, and a few pelvic adhesions were found. The ovaries were normal. Bilateral salpingectomy, right cornuectomy, ventrosuspension, and appendicectomy were performed. Histological examination showed evidence of tuberculous infiltration of the tubes, and a few acid-fast bacilli were demonstrated.

A course of streptomycin, 1 g. twice daily for 14 days, and para-aminosalicylic acid, $15 \mathrm{~g}$. daily, were prescribed. Radiological examination of the lungs and thoracic and lumbar vertebrae revealed no abnormality. No tubercle bacilli were isolated from the urine. The erythrocyte sedimentation rate was not raised. The patient was transferred to a convalescent home on May 3 .

\section{Discussion}

The frequency with which pregnancy occurs in the presence of pelvic tuberculosis is difficult to assess for the following reasons : (1) the incidence of unsuspected pelvic tuberculosis in apparently healthy females is unknown ; (2) pelvic tuberculosis may be first discovered after pregnancy has occurred; (3) in proved cases of pelvic tuberculosis pregnancy has rarely been reported.

\section{Incidence of Unsuspected Pelvic Tuberculosis}

The diagnosis of early cases of pelvic tuberculosis is notoriously difficult to make with certainty, even if the possibility is considered. The condition may be discovered at laparotomy or during investigations for sterility. The general impression obtained from surveying the literature is that pelvic tuberculosis is more common than is usually assumed. This fact is stressed by a number of writers, including Berkeley (1903), Sutherland (1943, 1950), Held (1947a, 1947b), Rabau (1950), and Halbrecht (1951).

\section{Pelvic Tuberculosis First Discovered after Pregnancy}

In such cases, as in the second patient described above, it remains uncertain which occurred first, the pregnancy or the pelvic tuberculosis. In the literature there are a number of records of patients who became pregnant and were found to have pelvic tuberculosis.

The Pregnancy may be Extrauterine.-Taylor (1910) stated that he had seen seven cases. Stevenson and Wharton (1939), Stein (1939), Bushby and Fisher (1940), Bland (1940), Hicks (1942), Shannon and Heller (1943), Mann and Meranze (1944), and Geisendorf (1950) describe single cases of an ectopic pregnancy in the presence of tuberculous salpingitis. Pink (1944) details the case record of a patient who had a second ectopic pregnancy two years after the first; tuberculous salpingitis was present on both occasions.

The Pregnancy may be Intrauterine.-Cooper in 1858 described the post-mortem findings of a case of rupture of the uterus, in the third month of pregnancy, from tuberculous degeneration of the fundus. Vineberg (1903) stated: "The occurrence of pregnancy in tuberculosis of the uterus is not an improbable event . . Thom, Schmorl, and Kockel have each reported a case of tuberculosis of the endometrium which had reached the stage of caseation, yet the pregnancy went to full term."
Abortion may Occur.-Wiseman and Retan (1927) described a case of abortion at the fifth month which was followed by severe post-abortive fever. The patient died four months later, the pyrexia not having subsided. At necropsy tuberculous endometritis, bilateral salpingitis, tuberculous peritonitis, and generalized miliary tuberculosis were found. Puxeddu (1940) described similar necropsy findings following an abortion.

A Viable Child may be Born.-A severe puerperal pyrexia may occur, leading to maternal death. Patoir, Decoulx, and Patoir (1937) described a maternal death in the puerperium. At necropsy advanced pelvic tuberculosis was found. Puxeddu (1940) reported the necropsy findings in two women, one dying in the puerperium and the other two months after confinement. In both cases advanced pelvic tuberculosis involving the uterus was found.

Jacob (1945) wrote about a new case of miliary tuberculosis in the puerperium, from genital tuberculosis. The patient gave birth to twins, who remained healthy. Severe puerperal pyrexia occurred and the mother died six weeks after delivery. At necropsy tuberculosis of the uterus and Fallopian tubes with generalized miliary tuberculosis was found. Georgescu et al. (1949) reported a case of maternal death on the ninth day of the puerperium. The baby had been delivered by forceps and puerperal pyrexia occurred. At necropsy pelvic tuberculosis and general miliary tuberculosis was found. The child died of bronchopneumonia at 3 months. Not all cases end as tragically as those just described. Herring and King (1950) described the case of an apparently healthy woman who had a normal confinement. Puerperal pyrexia developed, which settled only after she had received streptomycin. The mother and baby were discharged; both seemed fit. The baby died, when 22 days old, from miliary tuberculosis of the lungs. The mother was re-examined; no abnormality was found in the lungs. Tubercle bacilli were grown from the cervix. Tuberculous endometritis and cervicitis was diagnosed by curettage and biopsy. The patient was treated with streptomycin. Four months later no evidence of tuberculosis could be found on curettage.

In all these cases there was no concrete evidence that pelvic tuberculosis existed before pregnancy occurred. The assumption is that pelvic tuberculosis probably existed in a latent form.

\section{Pregnancy Rare in Patients with Pelvic Tuberculosis}

Fruhinsholz and Feuillade (1924), in their classical paper dealing with utero-adnexal tuberculosis and pregnancy, referred to cases reported by various observers of normal pregnancy following conservative surgery for pelvic tuberculosis. They described the case of a patient who had a baby when she was 21 . At 35 she had a conservative operation for pelvic tuberculosis, and after that had two abortions and then a normal delivery.

Pink (1944) recorded a second ectopic pregnancy in the presence of tuberculous salpingitis. Roulland (1947) described two cases of normal pregnancy and confinement following conservative operations for pelvic tuberculosis. Ewart Williams (1951) referred to a proved case of pelvic tuberculosis: the patient had been under the care of $\mathrm{Mr}$. J. Stallworthy, and had been treated with streptomycin and P.A.S. Subsequently she became pregnant.

\section{Summary and Conclusions}

Two cases are reported-one of the abortion of an intrauterine pregnancy in a patient with diagnosed pelvic tuberculosis, the other of pelvic tuberculosis discovered six months after a normal confinement.

A brief review of the literature dealing with pelvic tuberculosis and pregnancy is given.

The possibility of exacerbation of unsuspected pelvic tuberculosis following an apparently normal healthy pregnancy is emphasized. 
The frequency with which pregnancy occurs in the presence of pelvic tuberculosis is unknown. Probably many cases have not been reported because the diagnosis had not been established before pregnancy occurred. Recently a patient was seen in the antenatal clinic who four years previously had had bilateral tubal swellings which were thought to be tuberculous salpingitis. Should pregnancy occur it may be extrauterine, or, if intrauterine, abortion may ensue. There is suggestive evidence that unsuspected pelvic tuberculosis may rapidly progress in the presence of advanced pregnancy, possibly resulting in the death of the mother or infant, or both. To what extent modern antibiotics may alter the prognosis remains for future assessment.

I am indebted to Mr. C. M. Gwillim, who suggested reporting the first case, for his help, encouragement, and criticism in the preparation of this paper, and to $\mathrm{Mr}$. A. H. Charles for permission to report the second case.

\section{REFERENCES}

Berkeley, C. (1903). J. Obstet. Gynaec. Brit. Emp., 3, 31 Bland, P. B. (1940). Amer. J. Obstet. Gynec., 40, 271. Bushby, E. D., and Fisher, J. H. (1940). Ibid., 39, 125 Cooper, H. (1858). British Medical Journal, 847.

Fruhinsholz, A., and Feuillade, P. (1924). Gynéc. et Obstét., 10, 305

Geisendorf, W. (1950). Bull. Ass. Gynéc. Obstét. franc., 2, 388.

Georgescu, M., et al. (1949). Rev. franc. Gynéc., 44, 309.

Halbrecht, I. (1951). Lancet, 1, 85.

Held, E. (1947a). Gynaecologia, Basel, 123. 265

(1947b). Yearbook of Obstetrics and Gynecology, D. 426. Chicago. Herring, J. S., and King, J. A. (1950). Amer. J. Obstet. Gynec., 60, 925 Hicks, J. D. (1942). Med. J. Aust., 2, 240

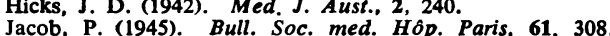

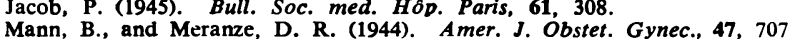
Mann, B., and Meranze, D. R. (1944). Amer. J. Obstet. Gynec., 47, 707
Patoir, A., Decoulx, P., and Patoir, G. (1937). Progr. méd., Parls, 64, 517. Pink, H. A. (1944), Amer. J. Obstet. Gynec., 48, 427.

Puxeddu, E. (1940). Int. Abstr. Surg., 71, 144.

Rabau, E. (1950). Fertil. and Steril., 1, 517

Roulland, M. H. (1947). C.R. Soc, franc Gynéc, 17, 145.

Shannon, D., and Heller, E. L. (1943). Amer. J. Obstet. Gynec., 45, 347.

Shannon, D., and Helle, E. Lo68.

Stein, I. F. (1939). Ibid., 38. 1068.

Stevenson. C. S., and Wharton, L. R. (1939). Ibid., 37, 303.

Sutherland. A. M. (1943). J. Obstet.

Taylor, A. M. (1910). Amer. J. Surg., 24, 173.

Vinebers, H. N. (1903). Trans. Amer. gynec. Soc., 28, 264.

Williams, E. (1951). Brittsh Medical Journal, 2, 52.

Wiseztan, J. R., and Retan, H. W. (1927). Arch. Intern. Med., 40, 65.

\section{Medical Memoranda}

\section{A Lithopaedion in Twins}

A married woman aged 29 first attended the surgery towards the end of July, 1950. Her period starting on June 14 had lasted only four days instead of the normal seven days, but the one before that had been at the beginning of May, making a six-weeks interval. Up to this time her periods had been quite regular. She was the mother of one child, then nearly 18 months old. After that confinement, which was perfectly normal, she had a retroverted uterus and a cervicitis for which cauterization was done. The uterus was replaced and a pessary inserted; but this failed to correct the retroversion, for which no further treatment was given, as it was not causing any symptoms.

At this time (July 18) it was presumed that she was five to six weeks pregnant and she was told to report again in six weeks' time unless there was any further trouble.

She next attended in the middle of September, when the uterus was found to be about the size of a 16-18 weeks' gestation. It was ascertained that there had been twins on the maternal side two generations previously, and an aunt of the father was also one of twins. The question then arose whether there were twins present, or whether the last period should be taken as the one at the beginning of May, either of which would fit in with the size of the uterus.
A month later the patient complained of pain low down in the right side. Examination revealed the uterus to be now almost to the umbilicus, with tenderness on the right side, about 2 in. $(5 \mathrm{~cm}$.) below McBurney's point. The B.P. was $110 / 70$ and the urine was negative, including a culture. The temperature, pulse, and respirations were normal. The patient was kept under observation, but, in view of the fact that there was no vaginal loss and the pain subsided gradually, nothing further was done at that time.

By the middle of December the uterus was a little less in size than one of 30 weeks. The foetal heart seemed to be normal and the position was R.O.A. The patient herself was very well. As the baby did not appear very large it was presumed that the period in June was the last normal one, and that the expected date of delivery would be March 21, 1951.

When she was examined at the end of January, 1951, the uterus was equivalent to one at 34 weeks and a breech presentation was found. The foetus was easily turned to a vertex position, and the head slipped into the pelvis very easily. Nothing abnormal was noted on vaginal examination. The baby still seemed to be on the small side, with a fair amount of amniotic fluid. $X$-ray examination showed one foetus, with no abnormality. The position was now R.O.A. The patient was seen again in one month, when everything seemed quite normal. The foetal heart was heard. The B.P. was $110 / 70$, the urine was clear, and the position was still R.O.A. with the head engaging.

As the patient had not been confined at the beginning of April, when she was two weeks overdue, a medical induction was carried out, and she went into labour immediately. While in labour a rectal examination revealed a ? prolapsed arm. A vaginal examination was done, and she was found to be three-quarters dilated, with a thin firm mass beside the head which felt like an arm, but was definitely not one. As there was plenty of room for the head, and the pains were strong and every two minutes, labour was allowed to continue under strict observation. A little over an hour later the patient was fully dilated. Coming down in front of the head was the mass which had felt like an arm and which was now found to be an almost completely calcified foetus, with the back presenting; the whole thing being curled round the occiput of the after-coming baby. No difficulties were presented, and a living female child of $5 \mathrm{lb}$. $4 \mathrm{oz}$. $(2.4 \mathrm{~kg}$.) was delivered.

Examination of the placenta revealed an infarction about the size of a duck's egg in the place where the cord of the lithopaedion arose. This calcified foetus was one of about four months, and the cause of the pain in the right side at about the fourth month could now be attributed to the infarction.

\section{COMMENT}

Masson and Simon (1928) state: "Lithopaedion is invariably the result of extrauterine pregnancy. It is extremely doubtful if a foetus retained within a normal uterus ever undergoes this transformation, because there the occurrence of infection leads rather to destruction of the tissues of the foetus and skeletonization." Cave (1937), reporting a case of lithopaedion, states that the pregnancy must be extrauterine. It is true that about half a dozen cases of intrauterine lithopaedion are reported in the very early literature ; but since none have appeared within the last hundred years their authenticity must be regarded with extreme scepticism.

It is to be assumed, therefore, that the above is an unusual case of one foetus, in a twin pregnancy, dying owing to an infarction or a small haemorrhage in the neighbourhood of the cord, and becoming almost completely calcified.

\section{J. KEELING RoberTS, M.R.C.S., L.R.C.P.}

\section{REFERENCES}

Cave, P. (1937). British Medical Journal, 1, 383.

Masson, J. C., and Simon, H. E. (1928). Surg. Gynec. Obstet., 46, 500. 